\title{
Research on Distribution Network Reliability Investment Estimation Method Based on Sequence Linearization Correlation Analysis
}

\author{
Huazhen $\mathrm{CAO}^{\mathrm{a}}$, Chong $\mathrm{GAO}^{\mathrm{a}}$, Yaxiong WU ${ }^{\mathrm{a}}$, Hao LI ${ }^{\mathrm{a}}$, Zijun $\mathrm{WANG}^{\mathrm{b}}{ }^{1}$, Chengmin \\ WANG $^{\mathrm{b}}$ \\ ${ }^{a}$ Grid Planning \& Research Center, Guangdong Power Grid Co. Guangzhou 510030, \\ China \\ ${ }^{b}$ Electronic Information and Electrical Engineering Department, Shanghai Jiaotong \\ University, Shanghai 200240, China
}

\begin{abstract}
This paper presents an estimation method of distribution network reliability planning Investment Based on sequence linearization correlation analysis. Firstly, the planning business index closely related to reliability are selected, and the control objectives of reliability index are decomposed into the promotion objectives of each planning business index through sequence linearization correlation analysis. Then, the typical engineering construction scenarios corresponding to each planning business index are constructed, and the investment required to achieve the corresponding promotion objectives of business index is estimated according to the typical scenarios, Finally, the total investment of reliability planning is obtained. The example shows that the method can be applied to the actual distribution network with complex grid conditions and various planning schemes, and can provide powerful guidance for power supply enterprises to improve the efficiency of capital use
\end{abstract}

Keywords. reliability, distribution network planning, correlation analysis, investment estimation

\section{Introduction}

With the continuous development of national economy, both industrial activities and people's living conditions require reliable power supply. Power supply reliability refers to the ability of power system to provide power and electricity to power users continuously according to acceptable quality standards and required quantity [1]. With the increasing attention to power supply reliability, researchers begin to study reliability planning. In the planning stage, methods to improve power supply reliability mainly include increasing liaison, increasing segmentation, optimizing grid structure, transformation of distribution automation, etc., which require huge capital investment.

${ }^{1}$ Corresponding author: Zijun Wang, Electronic Information and Electrical Engineering Department, Shanghai Jiaotong University, Shanghai 200240, China; Email: 505386064@qq.com. 
In order to control the cost, power supply companies must achieve a balance between reliability and economy. Reliability planning investment estimation has become an important part of decision-making in the background, which directly affects the capital plan and profit expectation of power supply enterprises.

In order to improve the effect of reliability planning, [2] considers the cluster island operation and studies the method of improving the reliability after DG access through two-step planning. Reference [3] applies the fuzzy set pair analysis method to multiobjective power grid planning, and [4] carries out active distribution network expansion planning based on uncertain network theory. Reference [5] presents a method to optimize the loop-based microgrid structure in an active distribution network. Reference [6] focus on contribution of wind farms to distribution network reliability. Reference [7] uses a minimal path based algorithm to compute the reliability of stochastic distribution network to balance reliability and delivery cost.

The existing studies have different explanations for the economy in reliability planning, but they rarely involve how to estimate the reliability investment. The investment data mostly come from post statistics, so it is difficult to estimate the future investment.

In terms of distribution network reliability index and its influencing factors, [8] studies the typical wiring mode of medium voltage distribution network, and [9] focuses on the optimal allocation of energy and analyzes the impact of various factors on reliability and economy. Reference [10] converts the reliability index of power grid into economic index, that is, puts forward the concept of "economic cost". Reference [11] [12] studies the reliability influencing factors in transmission network and hybrid microgrid, which has reference significance. Reference [13] presents the method to assess the reliability and risk implications of post-fault demand response. Reference [14] takes the influence of fault location and delay protection system into consideration. Reference [15] analyses urban distribution network through the application of a timesequential Monte Carlo simulation.

It can be found that the existing research results on distribution network reliability planning are not precise enough in the economic cost calculation of planning transformation, and can not be targeted to adapt to a variety of planning scenarios. In addition, the existing methods for obtaining the investment data of reliability planning largely rely on manual experience and post estimation, or simply make prediction based on the data of previous years. There lacks a theoretical model that can systematically and scientifically estimate the investment required in the planning scheme to improve the power supply reliability of medium voltage distribution network under specific scenarios.

In order to solve the problems above, this paper is committed to studying and calculating the planning investment estimation method required to achieve the given reliability index. By constructing a typical engineering construction scenario, this paper analyzes the engineering construction cost required for the improvement of each planning business index related to reliability on a micro scale, so as to provide more detailed and Reasonable reliability planning investment estimation method.

\section{Reliability Investment of Distribution Network}

The estimation problem of reliability planning investment is to estimate the planning investment required to achieve the predetermined reliability target. At present, the common practice is to build a data model for prediction according to the statistical value 
of reliability in recent years and the reliability planning investment in the corresponding year. Although this method is simple to implement, it has the following disadvantages: firstly, the lack of historical data samples will affect the estimation accuracy; Secondly, reliability planning investment does not represent the actual investment, and it often takes time to see the effect of reliability improvement after the transformationy, which leads to the weak correlation between the statistical value of reliability index and the reliability planning investment in the same year, thus affecting the estimation accuracy; Finally, some changes of management such as the change of reliability index statistical caliber and the adjustment of power supply jurisdiction, will also affect the estimation accuracy.

In order to deal with the above problems and give a more reasonable reliability planning investment estimation method, this paper presents an estimation method of distribution network reliability planning which is based on sequence linearization correlation analysis.

The business index managed by power companies refer to the key index of daily control of each business department. For example, the business index controlled by planning department include transferable power supply rate, cable utilization rate, feeder automation coverage rate, etc. Many business index are closely related to reliability, and business index are usually easier to count and manage. Therefore, power companies prefer to transform reliability index into business index, which is more intuitive and controllable. This paper is also based on business index. Firstly, the planning business index in correlation with reliability are selected, and then the reliability index is decomposed into the promotion targets of relevant planning business index. Then, by constructing a typical scenario for the promotion of each business index, the investment required for the promotion of each business index is estimated, and finally the total reliability planning investment is summarized. The overall idea is shown in Figure 1.

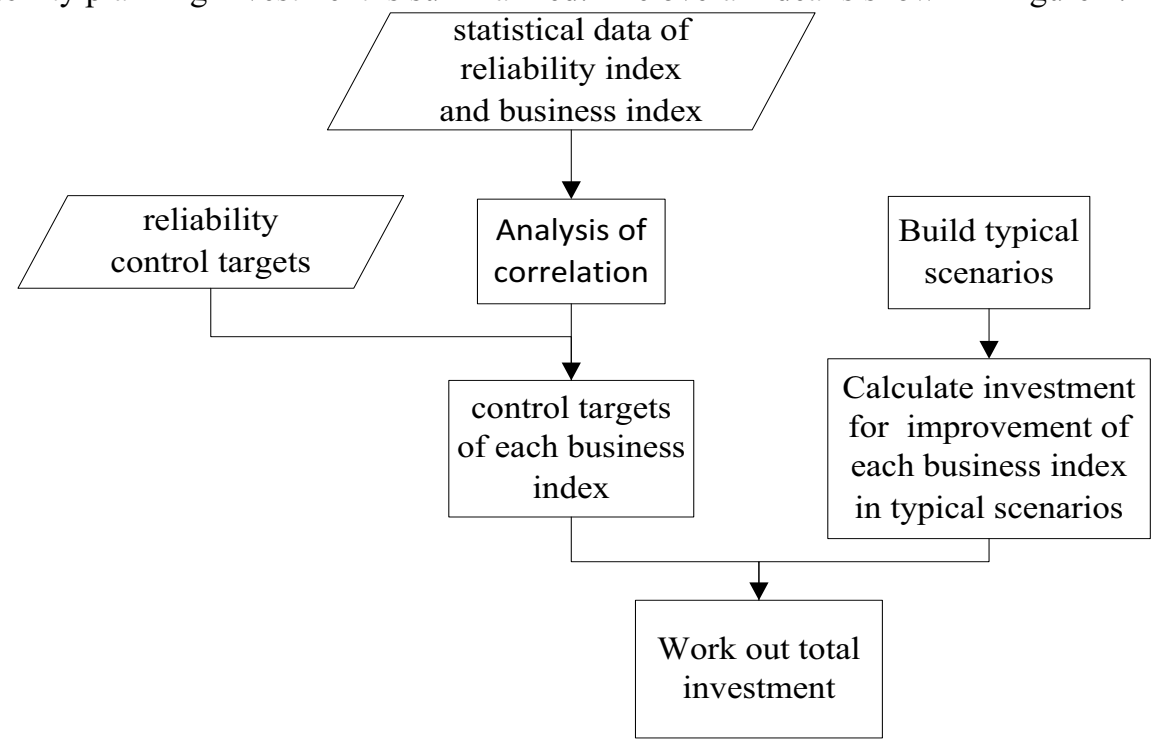

Figure 1. Investment estimation procedure for reliability planning.

The whole process is generally divided into two parts: business index decomposition and typical scenario estimation. 
In the business index decomposition stage, the first step is to collect the statistical data of reliability index and business index in historical years, and construct the correlation equation according to these data to obtain the correlation degree of each business index to the reliability index, and then decompose the reliability control targets into the control targets of each business index according to the correlation degree ranking of each business index.

In the typical scenario estimation stage, firstly typical scenarios for the improvement of various business index are built, then the average implementation cost of each typical scenario is estimated according to the collected distribution network project cost, the investment required for the improvement of various business index is worked out, and finally the total investment in reliability planning is summarized.

In the whole process, the overall reliability promotion target is transformed into the promotion target of a single business index, and then the promotion of a single business index is transformed into a typical planning scheme in combination with typical scenarios, so as to realize investment estimation. Compared with the common estimation methods, the calculation of this method is more precise and reasonable, and it is also more suitable for distribution network planning guided by business index.

\section{Analysis of Sequence Linearization Correlation}

When studying what transformation should be carried out to improve reliability index, it is necessary to determine the correlation between the improvement of various business index and the reliability index to be improved. The improvement of reliability index is affected by various business indexes, which is complex and diverse, and can be realized through the analysis of sequence linearization correlation degree.

Physical systems including power network are nonlinear, and the relationship between the performance of a nonlinear system and a state variable can be described mathematically as follows:

$$
y=f(x)
$$

where $\mathrm{x}$ and $\mathrm{y}$ represent the independent variable and dependent variable vector of the physical system respectively. The independent variable is generally the state variable of the physical system, and the dependent variable is generally the performance index of the physical system.

Considering the complexity of nonlinear systems, they are generally solved and analyzed by linearization or continuous linearization. Assuming that y changes only in a small neighborhood that can be linearized, there are:

$$
y=f\left(x_{0}\right)+f^{\prime}\left(x_{0}\right) \Delta x+\varepsilon\left(\Delta x^{2}\right)
$$

where $\varepsilon\left(\Delta x^{2}\right)$ represents terms other than primary and secondary after Taylor expansion. When these terms are ignored there are:

$$
\Delta y=f^{\prime}\left(x_{0}\right) \Delta x
$$


Written as a matrix:

$$
\Delta Y=A \Delta X
$$

where $\mathrm{A}$ is the incidence matrix, indicating the correlation degree between independent variables and dependent variables.

Generally, when it is difficult to establish a complete mathematical model of the physical system, the analysis method of big data can be used to fit the a-matrix. In this paper, assuming that $\mathrm{Y}$ represents the reliability investment cost of distribution network and $\mathrm{X}$ represents many factors affecting reliability, a matrix represents the degree of influence on reliability investment cost.

Since the above process is aimed at the linearization of nonlinear functions, it is necessary to determine the reference values $\mathrm{X}_{0}$ and $\mathrm{Y}_{0}$. Then, carry out big data analysis on this reference value. The independent variable and dependent variable are further corrected:

$$
x=x_{0}+\Delta x, y=y_{0}+\Delta y
$$

Therefore the modified values of the parameters to be solved of the incidence matrix are obtained. Then the corrected value is analyzed by big data, and the independent variable and dependent variable are corrected again. The $\mathrm{X}$ and $\mathrm{Y}$ values obtained from the solution are the change sequence from the reference value to the actual value. Repeat the correction process of the deviation until the values stop changing. This process is similar to the Newton method used in solving nonlinear equations.

Since the data of reliability parameters and various business index in the distribution network need to be updated frequently according to the monitoring values to ensure the latest state of safe and reliable operation of the system, it is usually not difficult to obtain a large number of statistical data. Through the correlation analysis of big data, the improvement of reliability index can be effectively linearized and decomposed into the improvement objectives of various business index.

In the stage of distribution network planning, the planning business index with great correlation with reliability are mainly four index: transferable power supply rate, cable utilization rate, average number of sections of overhead lines and feeder automation coverage. For the convenience of discussion, this paper only takes the most commonly used average outage time of users as the reliability index that should be paid attention to in the distribution network planning stage, and carries out the sequence linearization correlation analysis on this basis.

After collecting the statistical values of reliability index and business index in the last $3 \sim 5$ years, build a continuous linearized correlation equation as shown in equation (6).

$$
R_{t}=\sum_{j=1}^{n} K_{j} X_{t}^{j}+K_{0}
$$


where $R_{t}$ is the reliability index of year $\mathrm{t} ; X_{t}^{j}$ is the $\mathrm{j}$-th business index in year $\mathrm{t}$; $\mathrm{N}$ is the number of business index; $K_{j}$ is the linearization coefficient to be determined for the $\mathrm{j}$-th business index; $K_{0}$ is the coefficient to be determined for the constant term.

By plugging the data of $X_{t}^{j}$ and $R_{t}$ of each historical year (M years in total) into equation (6), the incidence matrix shown in equation (7) can be obtained.

$$
\left[\begin{array}{c}
R_{1} \\
R_{2} \\
\mathrm{M} \\
R_{m}
\end{array}\right]=\left[\begin{array}{lllll}
1 & X_{1}^{1} & X_{1}^{2} & \mathrm{~L} & X_{1}^{n} \\
1 & X_{2}^{1} & X_{2}^{2} & \mathrm{~L} & X_{2}^{n} \\
\mathrm{M} & & & & \mathrm{M} \\
1 & X_{m}^{1} & X_{m}^{2} & \mathrm{~L} & X_{m}^{n}
\end{array}\right]\left[\begin{array}{c}
K_{0} \\
K_{1} \\
K_{2} \\
\mathrm{M} \\
K_{n}
\end{array}\right]
$$

The sequence linearization correlation coefficient of each business index to the reliability index can be obtained by using the least square method. The higher the coefficient is, the more significant is the effect of the service index on reliability improvement. In other words, to improve reliability index, priority should be given to improving business index with high relevance.

The process of decomposing the reliability index control value (or planning value) into each business index control value (or planning value) according to the correlation degree of each business index to the reliability index can be expressed by the following equations.

$$
\left\{\begin{array}{l}
R_{m+1}-R_{m}=\sum_{j=1}^{n} K_{j}\left(X_{m+1}^{j}-X_{m}^{j}\right) \\
X_{m}^{j} \leq X_{m+1}^{j} \leq X_{\max }^{j}
\end{array}\right.
$$

where: $R_{m}$ is the statistical value of reliability index in the m-th year; $R_{m+1}$ is the control value of reliability index in the $m+1$ year; $X_{m}^{j}$ is the statistical value of the $\mathrm{j}$-th business index in the m-th year; $X_{m+1}^{j}$ is the statistical value of the $\mathrm{j}$-th business index in year $\mathrm{m}+1 ; X_{\max }^{j}$ is the upper limit of the $\mathrm{j}$-th business index.

According to the basic principle of distribution network and practical construction experience, the improvement of four index: transferable power supply rate, cable utilization rate, average number of sections of overhead lines and feeder automation coverage will enhance the reliability of power grid, resulting in the change of the control value of reliability index. For example, the value CAIDI (Customer Average Interruption Duration Index) discussed in this paper will decrease with the improvement of business index. CAIDI can be calculated as follows: 


$$
C A I D I=\frac{\sum_{i=1}^{N} \lambda_{i}}{N}
$$

where: $\lambda_{i}$ is is the total interruption time of the $\mathrm{i}$-th user; $N$ is the number of users.

In fact, the specific contribution of each index to reliability improvement is difficult to formulate through theoretical analysis. However, through the sequence linearization correlation analysis, the fine and reasonable linear correlation degree of business index based on big data can be obtained without a complete model of the reliability index affected by various factors. The fine and reasonable linear correlation degree of business index based on big data can be obtained, so as to transform the complex investment estimation problem into a linear optimization problem and guide the reliability planning of distribution network. The correlation coefficient between the reliability index and each business index obtained by solving the incidence matrix by the least square method reflects the impact of the change of each index on the change of reliability index.

\section{Investment Estimation Based on Typical Scenarios}

After decomposing the reliability index control value into control value of various business index, the cost required for the improvement of business index is estimated by constructing a typical scenario for the improvement of various business index, so as to obtain the planned investment required to achieve the reliability control objectives.

\subsection{Definition of Typical Scenarios}

While doing distribution network planning, different kinds of transformation are required to improve different business index. In this section, five typical scenarios that often occur in the actual construction and transformation of distribution network are defined. The construction and transformation forms of these typical scenarios are relatively standard and unified. After collecting information about engineering cost, more accurate investment estimates can be obtained. Specific scenarios are defined as:

Scenario 1: Adding a tie point to the existing overhead line. That is to add tie line and tie switch on the existing overhead single radiation line in order to transform it into hand-in-hand wiring mode. In this scenario the switchable power supply rate of distribution network is increased.

Scenario 2: Building a new line and adding a tie point. That is to build a new noload cable line as a public standby line, which is usually used to transform multiple single radiation lines into "n-1" main and standby wiring.

Scenario 3: Creating a new cable line. That is to build a new cable line in the area with low cable line coverage. In this scenario the cable utilization rate of distribution network is increased. This paper did not take the replacement of existing overhead lines with cable lines into consideration, mainly because except for the needs of municipal engineering, it is generally not allowed to directly change the existing overhead lines into cable lines in present. Optimization can only be performed on the basis of the existing grid. 
Scenario 4: Adding a section switch to the existing overhead line. That is to add a section switch to the existing overhead line. In this scenario the average number of sections of the overhead line of the distribution network is increased.

Scenario 5: Distribution automation transformation on existing lines. That is to erect intelligent switchgear and communication network for existing lines, namely distribution automation transformation. In this scenario the feeder automation coverage of distribution network is increased.

In the above five typical scenarios, scenario 1 and scenario 2 correspond to the improvement of switchable power supply rate, scenario 3 corresponds to the improvement of cable utilization rate, scenario 4 corresponds to the improvement of the average number of sections of overhead lines, and scenario 5 corresponds to the improvement of feeder automation coverage. Moreover, scenarios 1 to 4 are separately applied to the overhead network or cable network. Only scenario 5 covers the overhead network and cable network, which need to be discussed separately when estimating the cost.

\subsection{Investment Cost Calculation in Typical Scenarios}

The cost for the proposed five typical scenarios is calculated as follows.

Scenario 1: Adding a tie point to the existing overhead line

The planning scheme of adding a tie point to the existing overhead line can increase the transferable power supply rate of the distribution network. This scheme is applied to the project of transforming the single radiation network into a "2-1" single ring network.

The formula for investment cost in this scenario is

$$
C_{1}=L_{l}\left(c_{o}+r c_{o r}\right)+c_{o b}
$$

where $C_{1}$ is the cost of scenario $1 ; L_{l}$ is the length of the tie line, which usually takes $1 / 5 \sim 1 / 3$ of the power supply radius of the line; $c_{o}$ is the unit cost of overhead line; $\mathrm{R}$ is the construction coefficient, which is between 0 and $1 ; c_{o r}$ is the unit construction cost of overhead line erection (such as stringing and pole erection); $c_{o b}$ is the unit cost of polemounted switch.

Scenario 2: Building a new line and adding a tie point

The planning scheme of building a new line and adding a tie point can also increase the switchable power supply rate of the distribution network, but this scheme is applied to the project of transforming the single shot into the connection form of $\mathrm{N}$ supply and one standby. The formula for investment cost is as follows:

$$
C_{2}=L_{l}\left(c_{c}+r c_{c r}\right)+c_{c b}
$$

where $C_{2}$ is the cost of scenario $2 ; L_{l}$ is the length of the tie line; $c_{c}$ is the unit cost of cable; $\mathrm{R}$ is the construction coefficient; $c_{c r}$ is the unit construction cost of cable (such as trenching and landfilling); $c_{c b}$ is the unit cost of switchgear.

Scenario 3: Creating a new cable line 
The construction cost of the new cable line includes the cable conductor cost, switchgear cost, box transformer cost and construction cost estimated according to the average line length parameter. The specific calculation formula is

$$
C_{3}=L_{c}\left(c_{c}+r c_{c r}\right)+U c_{t}
$$

where $C_{3}$ is the cost of scenario $3 ; L_{c}$ is the length of new cable line; $c_{c}$ is the unit cost of cable; $\mathrm{R}$ is the construction coefficient; $c_{c r}$ is the unit construction cost of cable (such as trenching and landfilling); $\mathrm{U}$ is the average number of costumer, usually ranging from 15 to $35 ; c_{t}$ is the unit cost of distribution transformer.

Scenario 4: Adding a section switch to the existing overhead line

Adding a section switch to existing overhead lines can increase the average number of line sections, and the cost is related to the construction of section switches. The calculation formula is

$$
C_{4}=c_{o b}
$$

where $C_{4}$ is the cost of scenario $4 ; c_{o b}$ is the unit cost of pole-mounted switch.

Scenario 5: Distribution automation transformation on existing lines

Distribution automation transformation for existing lines can improve feeder automation coverage. It can be subdivided into two typical scenarios: overhead line polemounted switch transformation and cable line switch station transformation.

The investment cost for feeder automation transformation of an overhead line is

$$
C_{5 o}=\frac{1}{2} L_{o} r c_{o r}+2 c_{o b}
$$

where $C_{5 o}$ is the overhead line feeder automation transformation cost in scenario 5; $L_{o}$ is the total length of overhead line; $\mathrm{R}$ is the construction coefficient; $c_{o r}$ is the unit construction cost of overhead line feeder automation transformation (such as optical fiber erection); $c_{o b}$ is the unit cost of pole-mounted switch.

The investment cost for feeder automation transformation of a cable line is

$$
C_{5 c}=\frac{1}{2} L_{c} r c_{c r}+2 c_{c b}
$$

where $C_{5 c}$ is the cost of cable line feeder automation transformation in scenario 5; $L_{c}$ is the total length of the cable line; $\mathrm{R}$ is the construction coefficient; ${ }_{c r}$ is the unit construction cost for automation transformation of cable line feeder (such as optical fiber erection); ${ }^{c_{c b}}$ is the unit cost of switchgear. 


\subsection{Investment Cost Estimation}

After the cost of each typical scenario is calculated, the total investment is estimated according to the current value of each business index and the goal of reliability improvement. The key point of this process is to determine the engineering quantity of each typical scenario of overhead network and cable network.

Combining scenario 1 and scenario 2 we can get the investment estimation of increasing the renewable power supply rate as follows:

$$
C_{l}=C_{1} \Delta N_{o l}+C_{2} \Delta N_{c l}
$$

where $C_{l}$ is the investment cost for increasing the renewable power supply rate; $C_{1}$ and $C_{2}$ are the cost of scenario 1 and scenario 2 respectively; $\Delta N_{o l}$ and $\Delta N_{c l}$ are the number of overhead lines and cable lines that need to be connected.

Based on scenario 3, the investment estimate for increasing the cable utilization rate is

$$
C_{c}=k_{c} C_{3} \Delta N_{c}
$$

where $C_{c}$ is the investment cost for increasing cable utilization rate; $k_{c}$ is the conversion factor, since the addition of a cable is not only for improving the reliability of power supply but also for meeting the demand of load growth; $C_{3}$ is the cost of scenario 3; $\Delta N_{c}$ is the number of new cable lines.

Based on scenario 4, the investment estimate for increasing the average number of sections of overhead line is

$$
C_{s}=C_{4} N_{o}\left(X_{s}^{1}-X_{s}^{0}\right)
$$

where: $C_{s}$ is the investment cost of increasing the average number of sections of overhead line; $C_{4}$ is the cost of scenario $4 ; N_{o}$ is the number of overhead lines; $X_{s}^{1}$ is the target value of the average number of sections of overhead line; $X_{s}^{0}$ is the current value of the average number of sections of overhead line.

Based on scenario 5, the investment estimate for improving feeder automation coverage is

$$
C_{a}=C_{5 o} \Delta N_{o a}+C_{5 c} \Delta N_{c a}
$$

where: $C_{a}$ is the investment cost of improving feeder automation coverage; $C_{5 o}$ is the cost of implementing feeder automation for overhead lines in scenario $5 ; \Delta N_{o a}$ is the number of overhead lines requiring feeder automation; $C_{5 c}$ is the cost of implementing feeder automation for the cable line in scenario $5 ; \Delta N_{c a}$ is the number of cable lines that need to implement feeder automation. 
To sum up, $C_{l}+C_{c}+C_{s}+C_{a}$ is the total planned investment required to achieve the reliability control goal.

\section{Case Analysis}

This section takes the distribution network of a city as an example. The statistical values of the average outage time of costumers, various business index and the corresponding reliability planning investment in the last five years are shown in Table 1.

Table 1. Historical data of each index and investment in a city.

\begin{tabular}{cccccc}
\hline Year of data & $\mathbf{1}$ & $\mathbf{2}$ & $\mathbf{3}$ & $\mathbf{4}$ & $\mathbf{5}$ \\
\hline transferable power supply rate (\%) & 68.35 & 70.83 & 73.18 & 76.32 & 79.22 \\
cable utilization rate (\%) & 40.23 & 41.13 & 41.78 & 42.23 & 42.84 \\
average number of overhead & 2.65 & 2.74 & 2.81 & 2.88 & 2.94 \\
line sections & & & & & \\
feeder automation coverage (\%) & 8.34 & 15.23 & 31.78 & 50.44 & 68.23 \\
CAIDI (h/household) & 3.13 & 2.88 & 2.43 & 1.91 & 1.41 \\
Investmen (million yuan) & 0.83 & 0.94 & 1.15 & 1.38 & 1.59 \\
\hline
\end{tabular}

According to the four business index and the average outage time of costumer in each historical year in Table 1, the correlation matrix in the form of equation (7) is listed, and the correlation degree value shown in Table 2 is obtained through the sequence linearization correlation analysis.

Table 2. Correlation of business index and CAIDI in a city.

\begin{tabular}{cccc}
\hline transferable power supply rate & cable utilization rate & $\begin{array}{c}\text { average number of overhead } \\
\text { line sections }\end{array}$ & feeder automation coverage \\
\hline 1.83 & 0.66 & 11.09 & 1.31 \\
\hline
\end{tabular}

It is assumed that the planning goal is to reduce the average outage time of users from $1.41 \mathrm{~h} /$ household to $1.0 \mathrm{~h} /$ household. According to the correlation degree between each service index in Table 2 and the user average outage time index, the planning objectives of each service index can be obtained by solving equation group (8), as shown in Table 3. It can be seen from table 3 that the reliability control target can be met only by improving the two planned business index of transferable power supply rate and the average number of sections of overhead lines.

Table 3. Planning objectives of business index in a city.

\begin{tabular}{lccccc}
\hline & $\begin{array}{l}\text { transferable power } \\
\text { supply rate }(\%)\end{array}$ & $\begin{array}{l}\text { cable } \\
\text { rate } \\
(\%)\end{array}$ & $\begin{array}{l}\text { utilization } \\
(\%)\end{array}$ & $\begin{array}{l}\text { average number of } \\
\text { overhead } \\
\text { sections }\end{array}$ & $\begin{array}{c}\text { feeder automation } \\
\text { line }\end{array}$ \\
\hline Current value & 79.22 & & 42.84 & 2.94 & 68.23 \\
Target value & 86.72 & & 42.84 & 3.94 & 68.23 \\
\hline
\end{tabular}

By substituting the above planning improvement objectives into the actual project scenario, the number of overhead lines and cable lines that need to be connected can be obtained. See Table 4 for the number of lines and other calculation parameters required for investment estimation.

Table 4. Setting of other parameters (unit in million yuan per kilometre).

\begin{tabular}{cccc}
\hline$L_{l}$ & $c_{o}$ & $c_{o r}$ & $c_{o b}$ \\
\hline 2 & 56.65 & 9.86 & 7.05 \\
\hline
\end{tabular}




\begin{tabular}{cccc}
\hline $\mathbf{R}$ & $c_{c}$ & $c_{c r}$ & $c_{c b}$ \\
\hline 0.7 & 65.58 & 100.45 & 43.1 \\
\hline$L_{c}$ & $U$ & $c_{t}$ & $L_{o}$ \\
\hline 7.83 & 38.56 & 20.06 & 9.76 \\
\hline$N_{o}$ & $N_{c}$ & $\Delta N_{o l}$ & $\Delta N_{c l}$ \\
\hline 622 & 467 & 81 & 0 \\
\hline
\end{tabular}

According to equations (16) and (18), the investment amount required to improve the transferable power supply rate and the average number of sections of overhead lines in this calculation example can be calculated respectively. The planned investment cost required to achieve the reliability control goal is shown in Table 5.

Table 5. Estimation results of reliability planning investment (unit in 100 million yuan).

\begin{tabular}{ccccl}
\hline$C_{l}$ & $C_{c}$ & $C_{s}$ & $C_{a}$ & Total \\
\hline 1.09 & 0 & 0.44 & 0 & 1.53 \\
\hline
\end{tabular}

If the average outage time of costumer is reduced to $1.0 \mathrm{~h}$ per household according to the traditional regression prediction method, the investment required is about 189 million yuan. It can be seen that there is a certain deviation in the investment obtained by the two estimation methods, which is mainly due to the different principles of the two estimation methods. The estimation results of the method in this paper are greatly affected by the correlation analysis results of the four business index and the unit cost of equipment, while the estimation results of traditional methods are greatly affected by historical data. In this paper, the investment is allocated according to the idea of prioritizing the relevance of planning business index, so the investment amount obtained is more in line with the actual engineering scenario.

\section{Conclusion}

In this paper, a method for reliability planning investment estimation based on sequence linearization correlation analysis is proposed. According to the analysis results of the sequence linearization correlation between the distribution network planning business index and the reliability index, the planning targets of the reliability index are decomposed into the control targets of each business index, and then the typical scenarios for the improvement of each business index are constructed for investment estimation, so as to obtain a more reasonable reliability planning investment. This estimation method takes reliability and economy as a whole while considering the optimization of reliability improvement. It is a new exploration of distribution network planning and optimization. Moreover, this method can also be used to directly estimate the reliability planning investment according to the planning targets of each business index after the conventional planning of distribution network is completed.

In addition, for different districts and counties or different power supply districts under the same city, the reliability control objectives and promotion strategies will certainly be different. The correlation between each planned business index and reliability under each district and county or power supply district can be analyzed according to the core idea of this paper, and then solved according to the minimum total cost, Thus, a reliability planning model considering differentiated demand and optimal total investment is obtained. 


\section{References}

[1] Billinton R, Li W. Reliability assessment of electric power systems using monte Carlo methods [M]. New York: Plenum Press, 1994.

[2] Hu Di, Ding Ming, Bi Rui, et al. Two-step planning of grid-resource-storage based on cluster partition [J]. Automation of Electric Power System, 2020, 44(2):95-106.

[3] Jin Huazheng, Cheng Haozhong, Yang Xiaomei, et al. Application of fuzzy set pair analysis in multiobjective transmission network planning considering ATC $[J]$. Automation of Electric Power System, 2005, 29(21):49-53.

[4] Li Yan, Hu Zhijian, Zhang Menglin, et al. Active distribution network planning considering economy and reliability based on uncertain network theory[J]. Automation of Electric Power System, 2019, 43(16):6882.

[5] Cortes, C., Contreras, S., Shahidehpour, M, et al. Microgrid topology planning for enhancing the reliability of active distribution networks [J]. IEEE Transactions on Smart Grid, 2018, 9(6), 6369-6377.

[6] Blake, S., Taylor, P., Dent, C., Miller, D, et al. Quantifying the contribution of wind farms to distribution network reliability[J]. Wind Energy, 2014, 18(5), 941-953.

[7] Xu, X., Niu, Y., Song, Y, et al. Computing the reliability of a stochastic distribution network subject to budget constraint[J]. Reliability Engineering \& System Safety, 2021,216, 107947.

[8] Liu Xiangjun, MA Shuang, XU Gang. Formation of typical connection mode for distribution network by elementary connection model[J]. Power System Technology, 2012, 36(2):58-63.

[9] Liu Ziqiu, Hang Minxiang. Optimal energy allocation of microgrid based on reliability and economy by considering characteristics of micro source[J]. Power System Technology, 2014, 38(5):1352-1357.

[10] Zhu Xukai, Liu Wenyin, Yang Yihan. A new power network planning method by comprehensively considering system reliability factors[J]. Power System Technology, 2004, 28(21):51-54.

[11] Wang Yizhe, Tang Yong, Dong Chaoyang, et al. A hybrid criterion model for transmission system medium and long terms expansion planning[J]. Power System Technology, 2016, 40(7):2094-2098.

[12] Wei Yongzhong, Gu Ren, Yang Chen, et al. Optimization model for distribution network reliability investment considering interruptible load[J]. Smart Power, 2019, 47(2):107-112.

[13] Syrri, A., Mancarella, P. Reliability and risk assessment of post-contingency demand response in smart distribution networks[J]. Sustainable Energy, Grids and Networks, 2016, 7, 1-12.

[14] Xiao, F., Xia, Y., Zhou, Y, et al. Comprehensive reliability assessment of smart distribution networks considering centralized distribution protection system $[\mathrm{J}]$. IEEJ Transactions on Electrical and Electronic Engineering, 2019, 15(1), 40-50.

[15] Hernando-Gil, I., Ilie, I., Djokic, S, et al. Reliability planning of active distribution systems incorporating regulator requirements and network-reliability equivalents[J]. IET Generation, Transmission \& Distribution, 2016, 10(1), 93-106. 